\title{
Role of bile acids in the diagnosis and progression of liver cirrhosis: A prospective observational study
}

\author{
NING LIU ${ }^{1,2}$, JIAO FENG ${ }^{1}$, YANG LV ${ }^{1}$, QING LIU ${ }^{1}$, JINGFAN DENG ${ }^{1}$, \\ YUJING XIA $^{1}$, CHUANYONG GUO ${ }^{1}$ and YINGQUN ZHOU ${ }^{1}$ \\ ${ }^{1}$ Department of Gastroenterology, Shanghai Tenth People's Hospital, Tongji University, School of Medicine, Shanghai 200072; \\ ${ }^{2}$ The First Clinical Medical College of Nanjing Medical University, Nanjing, Jiangsu 210029, P.R. China
}

Received October 22, 2018; Accepted August 23, 2019

DOI: $10.3892 /$ etm.2019.8011

\begin{abstract}
The accumulation of toxic bile acids (BAs) is closely related to liver injury, inflammation and tumorigenesis. The aim of the present study was to determine the role of the serum BA spectrum in the diagnosis and progression of liver cirrhosis. This was a prospective observational study involving patients with chronic hepatitis $(n=23)$, liver cirrhosis $(n=101)$, and cirrhosis complicated with hepatocellular carcinoma (CC-HCC; $n=56$ ). The 6-month survival of cirrhotic patients was recorded after blood collection. Comparisons of serum total BAs and individual BAs between different groups were performed using the Mann-Whitney U or Kruskal-Wallis tests. Correlation analysis was conducted by Spearman's correlation. Diagnosis and prediction analyses were performed using receiver operating characteristic curves. Survival was analyzed using the Kaplan-Meier method and multivariable Cox regression analysis. The concentrations of total BAs, glycocholic acid (GCA), glycochenodeoxycholic acid (GCDCA), taurocholic acid (TCA), taurochenoxycholic acid and tauroursodeoxycholic acid (TUDCA) were increased significantly in patients with early cirrhosis compared to patients with chronic hepatitis $(\mathrm{P}<0.05)$ and were associated with the diagnosis of cirrhosis ( $\mathrm{P}=0.049,0.004,0.002,0.003,0.010$ and 0.009, respectively). The levels of total BAs, primary conjugated BAs, and TUDCA increased as liver cirrhosis progressed $(\mathrm{P}<0.05)$. Serum total BAs, GCA, GCDCA, and TCA predicted the 6-month survival of patients with liver cirrhosis $(\mathrm{P}=0.0003,0.005,0.002$, and 0.010 respectively). Based on multivariate Cox regression analysis, the level of total BAs was an independent predictor of mortality in cirrhotic patients (hazard ratios, 4.046; 95\% CI, 1.620-10.108; $\mathrm{P}=0.003)$. In the early-stage cirrhosis group, the
\end{abstract}

Correspondence to: Dr Yingqun Zhou, Department of Gastroenterology, Shanghai Tenth People's Hospital, Tongji University, School of Medicine, 301 Yanchang Middle Road, Shanghai 200072, P.R. China

E-mail: yqzh02@163.com

Key words: bile acids, liver cirrhosis, diagnosis, prognosis, hepatocellular carcinoma concentrations of total BAs and primary conjugated BAs were significantly elevated in patients with $\mathrm{CC}-\mathrm{HCC}$ compared with patients with cirrhosis alone. In conclusion, total and individual BAs, especially primary conjugated BAs, are effective non-invasive markers in the diagnosis and prognosis of liver cirrhosis, and may be potential indicators in the occurrence of hepatocellular carcinoma in patients with early cirrhosis.

\section{Introduction}

Liver cirrhosis is the end stage of liver disease and it has a number of different etiologies. Liver cirrhosis is currently the 11th most common cause of death worldwide (1). Hepatocellular carcinoma (HCC) is the most common cause of death in people with cirrhosis $(2,3)$. Although liver biopsy is the gold standard for diagnosing liver cirrhosis, it is not readily accepted by patients as it is an invasive procedure. No exact non-invasive markers have been identified to evaluate and monitor the progression of liver cirrhosis and the occurrence of HCC. Therefore, it is necessary to develop a safe, convenient, and effective method to monitor the occurrence and progression of liver cirrhosis.

Bile acids (BAs) are synthesized by a cascade of reactions catalyzed by enzymes acting upon hepatocyte cholesterol for recycling in the gut-liver axis (4). Factors including the gut microbiota (5), bile salt transporters (6) and nuclear receptor farnesoid-X-receptor (FXR) (7), participate in the regulation of BA homeostasis. Chronic cholestasis leads to fibrosis, cirrhosis, and eventually liver failure and hepatocellular/cholangiocellular carcinomas $(7,8)$. Recent studies have shown that alterations in BA homeostasis occur in liver diseases. The primary-to-secondary BA ratio is higher in non-alcoholic fatty hepatitis compared to healthy controls (9). glycocholic acid (GCA), taurocholic acid (TCA), glycochenodeoxycholic acid (GCDCA), taurochenoxycholic acid (TCDCA), and glycoursodeoxycholic acid (GUDCA) are significantly altered among different stages of hepatitis B-induced cirrhosis (10). In addition, higher concentrations of conjugated BAs, GCDCA and TCA are found in the serum of HCC patients compared with healthy controls (11). The elevated levels of conjugated BAs appear to be associated with early-stage HCC, whereas levels of BAs are elevated to a lesser extent in patients with more advanced HCC (11). These studies indicate the potential 
value of BAs as biomarkers for pathological progression in liver diseases; however, the role of BAs in the diagnosis and progression of liver cirrhosis has rarely been investigated.

Thus, it is hypothesized that total and individual BAs are sensitive indicators in assessing liver function and that studying the BA spectrum may provide a better understanding of the progression of liver cirrhosis and the occurrence of HCC. The aim of this present study was to investigate the linkage between serum BAs and the progression and prognosis of liver cirrhosis.

\section{Materials and methods}

Participants. Patients with chronic hepatitis $(n=23)$, cirrhosis $(\mathrm{n}=101)$ and cirrhosis complicated with HCC (CC-HCC; $\mathrm{n}=56$ ) hospitalized in Shanghai Tenth People's Hospital between January 2017 and January 2018 were enrolled into this prospective study. Healthy subjects $(n=22)$ were volunteer inpatients who had healthy examinations during the same period. Chronic hepatitis in this context referred to disease of the liver for $\geq 6$ months in duration, that had not developed into cirrhosis. Diagnosis of chronic hepatitis, cirrhosis or CC-HCC was established based on a detailed medical history; clinical signs; laboratory tests; imaging examinations; and liver biopsy, if necessary. The exclusion criteria were as follows: i) Patients who also presented with severe heart, brain, kidney or other organ disease; ii) patients with a history of gastrointestinal or hepatic surgery; iii) patients with a history of treatment with ursodeoxycholic acid (UDCA); iv) poor treatment compliance; v) patients who had had viral infections or hepatotoxicity medications during the preceding 6 months; and (6) patients who also presented with cholestasis caused by extrahepatic factors. Patients with HCC were also excluded from the cirrhosis group in this context. The Ethics Committee of Shanghai Tenth People's Hospital approved this study.

All patients provided a detailed medical history, underwent a physical examination, provided a blood sample, and underwent an enhanced abdominal CT scan. Liver function was assessed based on the Child-Pugh (CP) classification: Early-stage cirrhosis (CP A), middle stage cirrhosis (CP B) and late stage cirrhosis (CP C). The patients only received conservative and symptomatic treatment with medicine during the course of cirrhosis. Survival was recorded for 6 months after blood collection in cirrhotic patients. Model for end-stage liver disease (MELD) has been validated as an ideal and objective survival model in patients with chronic liver diseases $(12,13)$. It accurately predicts short-term mortality among patients with end-stage liver disease, and has been used worldwide for allocation of organs for liver transplantation (14). MELD scores were calculated using the following formula according to the guidelines of the United Network of Organ Sharing $(12,13)$ : MELD score $=9.57 \times \ln [$ creatinine $(\mathrm{mg} / \mathrm{dl})] 3.78 \times \ln$ [bilirubin $(\mathrm{mg} / \mathrm{dl})]+11.2 \times \ln (\mathrm{INR})+6.43$.

BA analysis. Blood samples were obtained from patients after an overnight fast. The serum was separated by centrifugation at $1,700 \mathrm{x}$ g for $10 \mathrm{~min}$ at $4^{\circ} \mathrm{C}$, and stored at $-80^{\circ} \mathrm{C}$ until the assay. Serum samples $(100 \mu \mathrm{l})$ were thawed and mixed with $500 \mu \mathrm{l}$ acetonitrile. After shaking vigorously for $5 \mathrm{~min}$ at room temperature and centrifugation at $14,000 \times \mathrm{g}$ for $5 \mathrm{~min}$ at $4^{\circ} \mathrm{C}, 400 \mu \mathrm{l}$ supernatant was dried with $60^{\circ} \mathrm{C}$ nitrogen and suspended with $100 \mu \mathrm{l}$ acetonitrile. The analysis of bile samples was performed on a Shimadzu high performance liquid chromatography apparatus (Shimadzu Corporation) coupled to an API3200 Triple-Quadrupole mass spectrometer (Applied Biosystems; Thermo Fisher Scientific, Inc.). Quantification was conducted using deuterated internal standards. BAs were separated using a Waters X SELECT column (3.0x50 mm; $3.5 \mu \mathrm{m}$; Waters Corporation) according to the manufacturer's instructions. Tandem mass spectrometry was operated in negative ion mode with multiple reaction monitoring (MRM) using settings in accordance with a previous study (15). The ion spray voltage was $-4,500 \mathrm{~V}$. The nitrogen gas temperature was $450^{\circ} \mathrm{C}$. The ion source gas 1 , ion source gas 2 and curtain gas were set at 40, 35 and 20 psi, respectively. The MRM transitions (m/z) were as follows: 407.3>407.3 for CA; 391.3>391.3 for UDCA, CDCA and DCA; 375.3>375.3 for LCA; 464.3>74 for GCA; 448.3>74 for GUDCA, GCDCA and GDCA; 432.3>74 for GLCA; 514.3>80 for TCA; 498.3>80 for TUDCA, TCDCA and TDCA; 482.3>80 for TLCA; $411.3>411.3$ for D4-CA; 395.3>395.3 for D4-CDCA, D4-DCA and D4-UDCA; 379.3>379.3 for D4-LCA; 468.3>74 for D4-GCA; 452.3>74 for D4-GCDCA, D4-GUDCA and D4-GDCA; 436.3>74 for D4-GLCA; 519.3>80 for D5-TCA; 503.3>80 for D5-TUDCA, D5-TCDCA and D5-TDCA; and 487.3>80 for D5-TLCA. The data obtained from the above platform were directly imported into Analyst software 1.6.2 (Applied Biosystems; Thermo Fisher Scientific, Inc.) for data pre-processing. Finally, the concentration of each substance was obtained by linear regression analysis also using Analyst software 1.6.2. The testing was performed in the Clinical Biochemical Laboratory of the Shanghai Tenth People's Hospital by experienced investigators.

The individual BA and the corresponding detection ranges were as follows: Cholic acid (CA), 20-2,000 nM; deoxycholic acid (DCA), 40-4,000 nM; chenodeoxycholic acid (CDCA), 40-4.000 nM; UDCA, 40-4,000 nM; lithocholic acid (LCA), 30-3,000 nM; GCA, 30-3,000 nM; glycolithocholic acid, 10-1,000 nM; glycodeoxycholic acid, 10-1,000 nM; GCDCA, 100-10,000 nM; GUDCA, 20-2,000 nM; TCA, 6-600 nM; taurolithocholic acid, 2-200 nM; taurodeoxycholic acid, 10-1,000 nM; TCDCA, 10-1,000 nM; and tauroursodeoxycholic acid (TUDCA), 5-500 $\mathrm{nM}$.

Statistical analysis. According to the distribution of the data, comparisons of continuous variables between groups were conducted using the one-way ANOVA (followed by a Bonferroni post hoc test), Kruskal-Wallis test (followed by a Dunn-Bonferroni post hoc test), unpaired Student's t-test or Mann-Whitney U test. Categorical variables were compared with the $\chi^{2}$ test or Fisher's exact test. Correlations between BAs and CP scores were computed using Spearman's correlation analysis. Diagnosis and prediction analyses were determined by receiver operating characteristic (ROC) curves. Cut-off values were determined via the Youden index. The Kaplan-Meier method was conducted to analyze survival, and comparisons were made with the log-rank test. A Cox regression analysis was used to analyze the association between BAs and mortality, and the association was adjusted for age and MELD score. Statistical analyses were carried out using SPSS version 
Table I. Clinical information and characteristics of patients with chronic hepatitis and cirrhosis.

\begin{tabular}{|c|c|c|c|c|}
\hline Parameter & Chronic hepatitis $(n=23)$ & $\mathrm{CPA}(\mathrm{n}=38)$ & CP B $(n=32)$ & $\mathrm{CPC}(\mathrm{n}=31)$ \\
\hline Age, years & $63 \pm 8$ & $62 \pm 9$ & $65 \pm 12$ & $65 \pm 7$ \\
\hline $\operatorname{Sex}, \mathrm{M} / \mathrm{F}$ & $15 / 8$ & $25 / 13$ & $22 / 10$ & $21 / 10$ \\
\hline \multicolumn{5}{|l|}{ Etiology } \\
\hline Viral, n (\%) & $11(47.8)$ & $18(47.4)$ & $17(53.1)$ & $16(51.6)$ \\
\hline Alcoholic, n (\%) & $5(21.7)$ & $8(21.1)$ & $5(15.6)$ & $6(19.4)$ \\
\hline Autoimmune, n (\%) & $3(13.0)$ & $4(10.5)$ & $4(12.5)$ & $3(9.7)$ \\
\hline Others, n $(\%)$ & $4(17.4)$ & $8(21.1)$ & $6(18.8)$ & $6(19.4)$ \\
\hline ALT, U/1 & $68 \pm 21$ & $25 \pm 18^{a}$ & $23 \pm 20$ & $26 \pm 14$ \\
\hline ALP, U/1 & $80 \pm 33$ & $86 \pm 30$ & $97 \pm 42$ & $128 \pm 51^{\mathrm{c}}$ \\
\hline Albumin, $\mathrm{g} / \mathrm{l}$ & $41 \pm 3$ & $40 \pm 4$ & $29 \pm 6^{b}$ & $26 \pm 4^{c}$ \\
\hline PT, sec & $12.9 \pm 1.1$ & $13.1 \pm 1.4$ & $14.7 \pm 1.5$ & $17.1 \pm 3.6^{\mathrm{c}}$ \\
\hline
\end{tabular}

${ }^{\mathrm{a}} \mathrm{P}<0.05$ vs. patients with chronic hepatitis; ${ }^{\mathrm{b}} \mathrm{P}<0.05$ vs. patients with $\mathrm{CP} \mathrm{A} ;{ }^{\mathrm{c}} \mathrm{P}<0.05$ vs. patients with $\mathrm{CP} \mathrm{B}$. ALT, alanine transaminase; $\mathrm{ALP}$, alkaline phosphatase; CP, Child-Pugh score; CP A, early stage cirrhosis; F, female; M, male; PT, prothrombin time.

20.0 (IBM Corp.) and GraphPad Prism software version 7.0 (GraphPad Software, Inc.) for Windows. P<0.05 was considered to indicate a statistically significant difference. Partial least squares-discriminant analysis (PLS-DA) was used to visualize the BA metabolome, which was conducted using the MetaboAnalyst 4.0 tool (http://www.metaboanalyst.ca/) (16).

\section{Results}

Baseline characteristics. Participants enrolled in the study were 40-80 years of age. Cirrhotic patients included 51 (50.5\%) with viral hepatitis cirrhosis, 19 (18.8\%) with alcoholic liver cirrhosis, $11(10.9 \%)$ with autoimmune hepatitis cirrhosis, and $20(19.8 \%)$ with cirrhosis due to other etiologies. The cirrhotic patients were separated into three groups based on the $\mathrm{CP}$ classification (CP A, CP B, and CP C), with 38 (37.6\%), 32 $(31.7 \%)$, and $31(30.7 \%)$ patients, respectively. Patients with chronic hepatitis included $11(47.8 \%)$ with viral hepatitis, 5 (21.7\%) with alcoholic hepatitis, $3(13.0 \%)$ with autoimmune hepatitis, and $4(17.4 \%)$ due to other etiologies. No statistically significant differences in age, sex, and etiology existed in patients with chronic hepatitis and CP A, as well as patients with different stages of cirrhosis (Table I). During the 6-month follow-up, 24 (23.8\%) cirrhotic patients died. Age and sex did not differ statistically between the survival and death groups (Table II). There was no significant difference in age and sex between the healthy subjects, patients with cirrhosis, and patients with CC-HCC (Table III).

BAs in patients with chronic hepatitis and cirrhosis. Patients with cirrhosis had significant elevations in the concentrations of primary conjugated BAs (GCA, GCDCA, TCDCA and TCA), TUDCA and total BAs compared with chronic hepatitis patients $(\mathrm{P}<0.05$; Figs. 1 and 2$)$. The ROC curves revealed that GCDCA had the best diagnostic performance for liver cirrhosis $(0.735 ; \mathrm{P}=0.002)$, followed by TCA $(0.726 ; \mathrm{P}=0.003)$, GCA (0.719; $\mathrm{P}=0.004)$, TUDCA (0.701; $\mathrm{P}=0.009)$, TCDCA (0.697; $\mathrm{P}=0.010)$, and total BAs $(0.652 ; \mathrm{P}=0.049)$, with an area
Table II. Clinical information of cirrhotic patients in the survival and death groups (6 months following blood sample collection).

\begin{tabular}{lccr}
\hline Parameter & $\begin{array}{c}\text { Survival } \\
(\mathrm{n}=77)\end{array}$ & $\begin{array}{c}\text { Death } \\
(\mathrm{n}=24)\end{array}$ & P-value \\
\hline Age, years & $63 \pm 12$ & $68 \pm 10$ & 0.070 \\
Sex, M/F & $51 / 27$ & $17 / 6$ & 0.614 \\
Etiology & & & \\
Viral, n $(\%)$ & $40(51.9)$ & $11(45.8)$ & 0.601 \\
Alcoholic, n $(\%)$ & $15(19.5)$ & $4(16.7)$ & 0.993 \\
Autoimmune, n $(\%)$ & $8(10.4)$ & $3(12.5)$ & 0.720 \\
Others, n $(\%)$ & $14(18.2)$ & $6(25.0)$ & 0.558 \\
ALT, U/l & $21 \pm 16$ & $25 \pm 23$ & 0.916 \\
ALP, U/1 & $95 \pm 38$ & $129 \pm 55$ & 0.001 \\
Albumin, g/1 & $34 \pm 8$ & $28 \pm 5$ & 0.002 \\
PT, sec & $14.6 \pm 2.6$ & $16.6 \pm 4.3$ & 0.012 \\
MELD & $12.34 \pm 4.73$ & $17.19 \pm 5.32$ & $<0.001$ \\
& & &
\end{tabular}

ALT, alanine transaminase; ALP, alkaline phosphatase; F, female; M, male; PT, prothrombin time; MELD, model for end-stage liver disease.

under the receiver operating characteristic curve (AUROC) of 0.735 [95\% confidence interval (CI), 0.605-0.864], 0.726 (95\% CI, 0.601-0.851), 0.719 (95\% CI, 0.591-0.847), 0.701 (95\% CI, $0.566-0.837), 0.697$ (95\% CI, 0.565-0.829), and 0.652 (95\% CI, 0.515-0.788), respectively (Fig. 3).

BAs in patients with liver cirrhosis. The level of total BAs significantly increased as liver cirrhosis progressed (Fig. 2). The concentrations of primary conjugated BAs and TUDCA were significantly higher in the $\mathrm{CP} \mathrm{B}$ and $\mathrm{CP} \mathrm{C}$ groups than in the CP A group $(\mathrm{P}<0.05)$. The secondary BAs (DCA and 
Table III. Clinical information of healthy subjects, patients with cirrhosis and CC-HCC.

\begin{tabular}{|c|c|c|c|}
\hline Parameter & Healthy control $(n=22)$ & Cirrhosis $(n=101)$ & $\mathrm{CC}-\mathrm{HCC}(\mathrm{n}=56)$ \\
\hline Age (years) & $63 \pm 9$ & $64 \pm 9$ & $63 \pm 8$ \\
\hline Gender (M/F) & $15 / 7$ & $68 / 33$ & $40 / 16$ \\
\hline Child-Pugh A/B/C & N/A & $38 / 32 / 31$ & $15 / 23 / 18$ \\
\hline \multicolumn{4}{|l|}{ Etiology } \\
\hline Viral, n (\%) & N/A & $51(50.5)$ & $29(51.8)$ \\
\hline Alcoholic, n (\%) & $\mathrm{N} / \mathrm{A}$ & $19(18.8)$ & $10(17.9)$ \\
\hline Autoimmune, n (\%) & N/A & $11(10.9)$ & $4(7.1)$ \\
\hline Others, n $(\%)$ & N/A & $20(19.8)$ & $13(23.2)$ \\
\hline $\operatorname{ALT}(\mathrm{U} / \mathrm{l})$ & $16 \pm 7$ & $25 \pm 18^{\mathrm{b}}$ & $48 \pm 39^{a}$ \\
\hline $\operatorname{ALP}(\mathrm{U} / \mathrm{l})$ & $68 \pm 19$ & $103 \pm 45$ & $125 \pm 62^{\mathrm{a}}$ \\
\hline Albumin (g/l) & $43 \pm 6$ & $33 \pm 8^{\mathrm{a}}$ & $31 \pm 8^{\mathrm{a}}$ \\
\hline PT (s) & $10.9 \pm 0.8$ & $15.0 \pm 3.1^{\mathrm{a}}$ & $14.5 \pm 3.0^{\mathrm{a}}$ \\
\hline \multicolumn{4}{|l|}{ Clinical characteristics } \\
\hline Ascites & N/A & $58(57.4)$ & $34(60.7)$ \\
\hline Spontaneous bacterial peritonitis & N/A & $13(12.9)$ & $3(5.4)$ \\
\hline Hepatic encephalopathy & N/A & $24(23.8)$ & $6(19.7)$ \\
\hline \multicolumn{4}{|l|}{ Medical history } \\
\hline Upper gastrointestinal hemorrhage & N/A & $51(50.5)^{\mathrm{b}}$ & $18(32.1)$ \\
\hline Symptomatic treatment of medicine & $\mathrm{N} / \mathrm{A}$ & $101(100)$ & $56(100)$ \\
\hline
\end{tabular}

${ }^{a} \mathrm{P}<0.05$, patients with cirrhosis (or CC-HCC) vs. healthy controls; and ${ }^{\mathrm{b}} \mathrm{P}<0.05$, patients with cirrhosis vs. patients with CC-HCC. ALT, alanine transaminase; ALP, alkaline phosphatase; CC-HCC, cirrhosis complicated with hepatocellular carcinoma; F, female; M, male; N/A, not applicable; PT, prothrombin time.
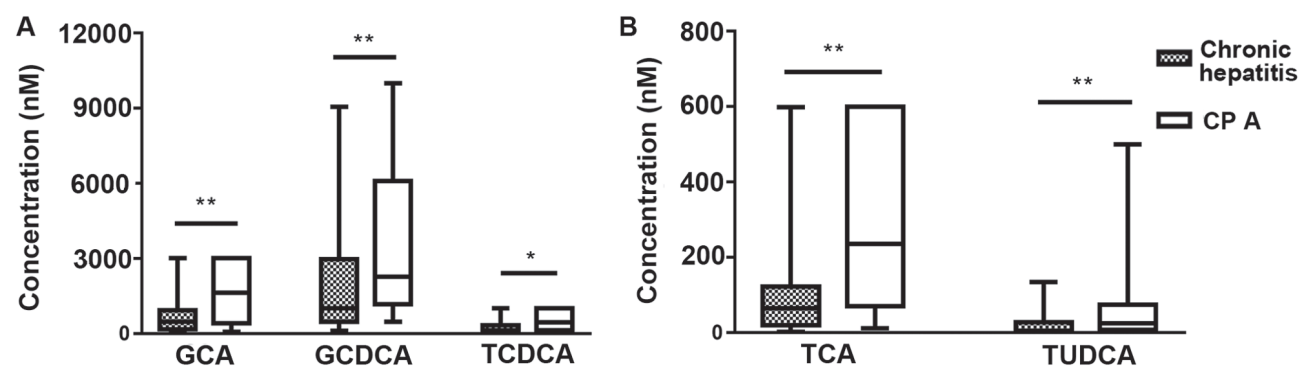

Figure 1. Box diagram of individual serum bile acids in patients with chronic hepatitis and CP A patients. (A) Comparison of GCA, GCDCA, and TCDCA concentrations. (B) Comparison of TCA and TUDCA concentrations. "P $<0.05,{ }^{* * *} \mathrm{P}<0.01$ (Mann-Whitney U test). CP, Child Pugh score; CP A, early stage cirrhosis; GCA, glycocholic acid; GCDCA, glycochenodeoxycholic acid; TCA, taurocholic acid; TCDCA, taurochenoxycholic acid; TUDCA, tauroursodeoxycholic acid.

LCA) decreased with the severity of liver disease, but there was no statistical significance (Fig. 4). Spearman correlation analysis showed that the level of total BAs was significantly correlated with the $\mathrm{CP}$ score $(\mathrm{r}=0.580, \mathrm{P}<0.001)$. Among the individual BAs, the concentrations of GCA, GCDCA, TCA, TCDCA, and TUDCA were positively correlated with CP scores [Spearman r (GCA, 0.489, P<0.001; GCDCA, 0.520, $\mathrm{P}<0.001$; TCA, 0.545, $\mathrm{P}<0.001$; TCDCA, 0.571, $\mathrm{P}<0.001$; TUDCA, 0.467, P<0.001); Table IV].

Cirrhotic patients were analyzed as two groups, depending on their survival after 6 months from blood sample collections. Total BAs, GCA, GCDCA, TCA and TCDCA showed a significant increase in the death group $(\mathrm{P}<0.05$; Figs. 2 and 5). Because the levels of GCA, TCA and TCDCA increased above the maximum detection range in the majority of deceased patients, the shapes of the box plots looked unusual in Fig. 5 (if the levels of BAs exceed the maximum detection range, the samples can be diluted to obtain precise values; however, considering the limitation of the sample volume, the samples were not diluted to retest the specific levels of individual BAs). The concentrations of total BAs, GCA, GCDCA, and TCA were statistically significant in predicting the 6-month survival in cirrhotic patients $(\mathrm{P}=0.0003,0.005,0.002,0.010$ respectively), with an AUROC of 0.744 (95\% CI, 0.616-0.872), 0.689 (95\% CI, 0.583-0.796), 0.707 (95\% CI, 0.592-0.823), and 0.674 (95\% CI, 0.565-0.784) respectively (Fig. 6). MELD score is well known for its good 


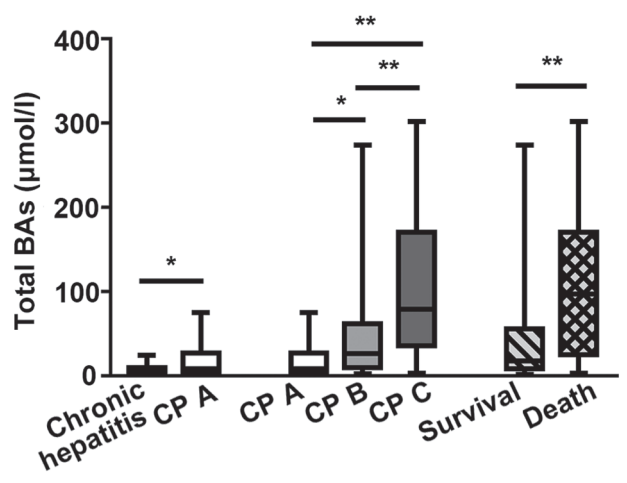

Figure 2. Box diagram of serum total BAs in the different $\mathrm{CP}$ groups of patients. ${ }^{*} \mathrm{P}<0.05,{ }^{* * *} \mathrm{P}<0.01$ [Kruskal-Wallis (followed by Dunn-Bonferroni post hoc method) or Mann-Whitney U test]. BA, bile acid; CP, Child-Pugh score; CP A, early stage cirrhosis; $\mathrm{CP} \mathrm{B}$, middle stage cirrhosis; $\mathrm{CP} \mathrm{C}$, late stage cirrhosis.

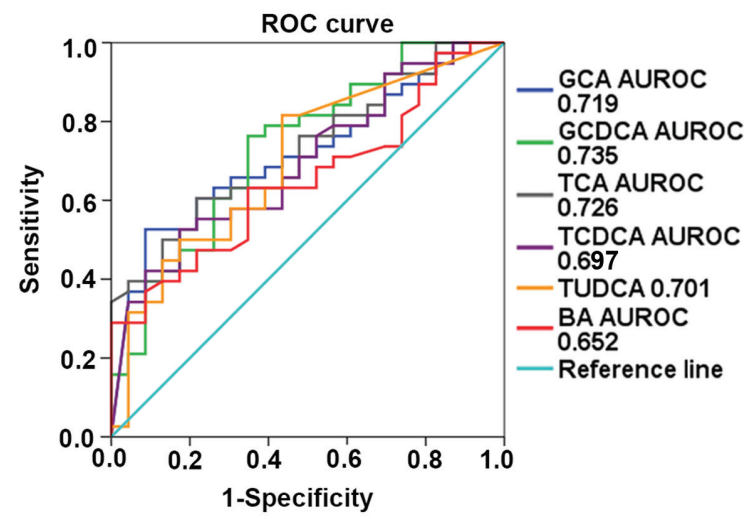

Figure 3. ROC curves of total BAs and five individual BAs for diagnosing early cirrhosis $(\mathrm{P}<0.05)$. AUROC, area under the receiver operating characteristic curve; BA, bile acid; GCA, glycocholic acid; GCDCA, glycochenodeoxycholic acid; ROC, receiver operating characteristic; TCA, taurocholic acid TCDCA, taurochenoxycholic acid; TUDCA, tauroursodeoxycholic acid.

performance in evaluating survival of patients with end-stage cirrhosis (13). In this present study, MELD score strongly predicted the 6-month survival of cirrhotic patients $(\mathrm{P}<0.001)$, with an AUROC of 0.773 (95\% CI, 0.676-0.870). Although the prediction accuracies of BAs were slightly lower than those of the MELD score, BAs were still effective and convenient prognostic indicators for cirrhotic patients. The Youden index revealed total BAs $\geq 75.7 \mu \mathrm{mol} / 1$, GCA $\geq 2,954 \mathrm{nmol} / 1$, GCDCA $\geq 8,400 \mathrm{nmol} / 1, \mathrm{TCA} \geq 599.5 \mathrm{nmol} / 1$, and TCDCA $\geq 619.5 \mathrm{nmol} / 1$ as best cut-off values. The cumulative 6-month survival was significantly higher in patients with total $\mathrm{BAs} \geq 75.7 \mu \mathrm{mol} / 1$ in comparison with total BAs $<75.7 \mu \mathrm{mol} / 1(\mathrm{P}<0.001)$. The mortality of patients with a high concentration of GCA, TCA, GCDCA, or TCDCA was significantly higher than that of patients with low concentrations of GCA, GCDCA, TCA or TCDCA ( $\mathrm{P}=0.004,0.001,0.005$ and 0.021 , respectively; Fig. 6). The present study further analyzed whether BAs were still a relevant predictor of mortality after adjustment for age and MELD score by using Cox regression. This showed that total BAs $\geq 75.7 \mu \mathrm{mol} / 1$ (hazard ratio, 4.046; 95\% CI, 1.620-10.108; $\mathrm{P}=0.003$ ) significantly predicted mortality in cirrhotic patients independently of age and MELD score (Table V). A high level of
Table IV. Correlations of Child-Pugh scores with BAs by Spearman analysis.

\begin{tabular}{lcc}
\hline BAs & Correlation coefficient & P-value \\
\hline Total BAs & 0.580 & $<0.001$ \\
GCA & 0.489 & $<0.001$ \\
GCDCA & 0.520 & $<0.001$ \\
TCA & 0.545 & $<0.001$ \\
TCDCA & 0.571 & $<0.001$ \\
TUDCA & 0.467 & $<0.001$ \\
\hline
\end{tabular}

BAs, bile acids; GCA, glycocholic acid; GCDCA, glycochenodeoxycholic acid; TCA, taurocholic acid; TCDCA, taurochenoxycholic acid; TUDCA, tauroursodeoxycholic acid.

total BAs was associated with a poor outcome among cirrhotic patients. However, after adjustment for age and MELD, the individual BAs were no longer significant predictors for mortality (data not shown).

BAs in patients with liver cirrhosis and CC-HCC. PLS-DA was used to visualize the BA metabolism in healthy subjects, patients with liver cirrhosis and patients with CC-HCC. Patients with liver cirrhosis and CC-HCC were distinctly separated from healthy subjects, but the clusters showed little variation between patients with cirrhosis and CC-HCC (Fig. 7). Levels of total and individual BAs were analyzed in patients with cirrhosis and CC-HCC with the same CP class. In the early-stage (CP A), the concentration of total BAs was significantly higher in patients with CC-HCC compared with patients with cirrhosis [median, $22 \mu \mathrm{mol} / 1$; inter-quartile range (IQR), 10.4-32.6 vs. median, $8.7 \mu \mathrm{mol} / \mathrm{l}$; IQR, 4.2-26.5 respectively; $\mathrm{P}=0.049$ ], and the levels of primary conjugated BAs, and TUDCA were significantly higher in patients with CC-HCC compared to patients with cirrhosis (Fig. 8). In the advanced stage, the concentrations of BAs were not significantly different between the two groups (data not shown). In patients with $\mathrm{CC}-\mathrm{HCC}$, Spearman correlation analysis showed no significant correlation between the levels of total and individual BAs and Barcelona scores. During the 6-month follow-up, $23(41.1 \%)$ patients died. There was no significant difference in the levels of total BAs between the blood samples collected from patients who survived compared to patients who died (median, $31.7 \mu \mathrm{mol} / \mathrm{l}$; IQR 10.4-64.5 vs. median, $63.4 \mu \mathrm{mol} / 1$; IQR, 20.5-109.9 respectively; $\mathrm{P}=0.067$ ). The individual BAs also showed no significant differences among the deceased and surviving patients (Fig. S1).

\section{Discussion}

A previous study indicated the correlation between serum total BA levels and mortality risk in patients with cirrhosis (17). Portal-systemic shunting (18) and diminished hepatic clearance of BAs (19) are thought to be two of the most essential factors in the elevation of serum BAs in patients with cirrhosis. Recent studies have shown that alterations in the intestinal flora play an indispensable role in BA disorders among cirrhotic patients $(20,21)$. 
Table V. Cox regression analysis for 6-month mortality.

\begin{tabular}{lccc}
\hline Variables & $\begin{array}{c}\text { Univariate Cox } \\
\text { regression HR (95\% CI) }\end{array}$ & P-value & $\begin{array}{c}\text { Multivariate Cox } \\
\text { regression HR (95\% CI) }\end{array}$ \\
\hline Age, years & $1.045(0.996-1.096)$ & 0.074 & $1.047(0.986-1.112)$ \\
MELD score & $1.154(1.073-1.242)$ & $<0.001$ & $1.092(1.005-1.187)$ \\
Total BAs $\geq 75.7 \mu \mathrm{mol} / 1$ & $5.998(2.656-13.548)$ & $<0.001$ & $4.046(1.620-10.108)$ \\
\hline
\end{tabular}

BAs, bile acids; CI, confidence intervals; HR, hazard ratios; MELD, model for end-stage liver disease.
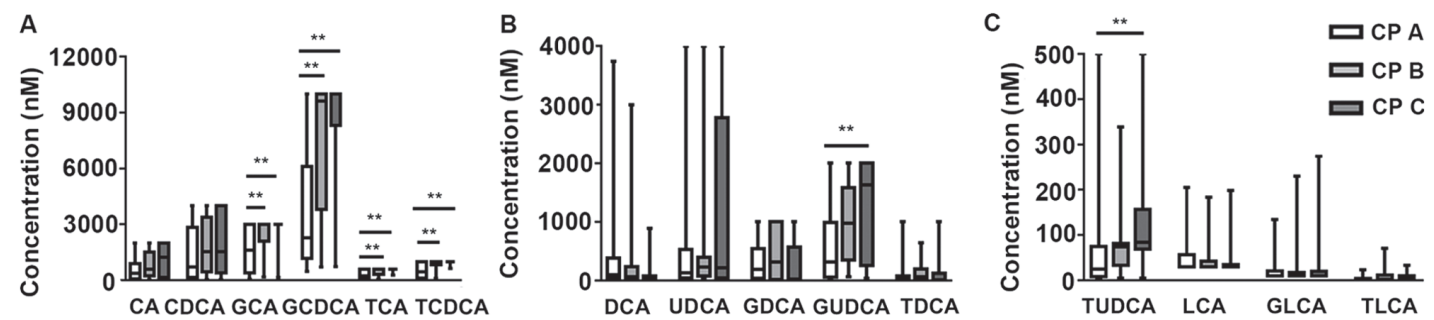

Figure 4. Box diagrams of individual serum bile acids in different stages of $\mathrm{CP}$ classification in cirrhotic patients. (A) Comparison of CA, CDCA, GCA, GCDCA, TCA, and TCDCA concentrations. (B) Comparison of DCA, UDCA, GDCA, GUDCA, and TDCA concentrations. (C) Comparison of TUDCA, LCA, GLCA, and TLCA concentrations. ${ }^{* *} \mathrm{P}<0.01$ [Kruskal-Wallis test (followed by Dunn-Bonferroni post hoc method)]. CA, cholic acid; CDCA, chenodeoxycholic acid; CP, Child-Pugh score; CP A, early stage cirrhosis; CP B, middle stage cirrhosis; CP C, late stage cirrhosis; DCA, deoxycholic acid; GCA, glycocholic acid; GCDCA, glycochenodeoxycholic acid; GLCA, glycolithocholic acid; GUDCA, glycoursodeoxycholic acid; LCA, lithocholic acid; TCA, taurocholic acid; TCDCA, taurochenoxycholic acid; TDCA, taurodeoxycholic acid; TLCA, taurolithocholic acid; TUDCA, tauroursodeoxycholic acid; UDCA, ursodeoxycholic acid.

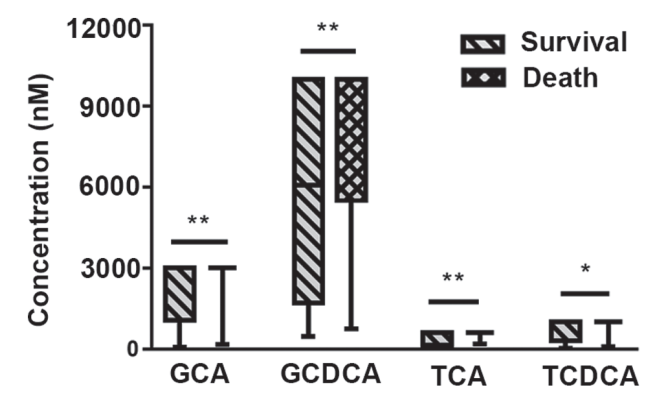

Figure 5. Box diagrams of 4 individual BAs in the survival and death groups during the 6-month follow-up. ${ }^{*} \mathrm{P}<0.05,{ }^{* * *} \mathrm{P}<0.01$ (Mann-Whitney $\mathrm{U}$ test). The unusual shape of the box plots was due to levels of BAs exceeding the maximum detection range. GCA, glycocholic acid; GCDCA, glycochenodeoxycholic acid; TCA, taurocholic acid; TCDCA, taurochenoxycholic acid.

Furthermore, FXR plays a pivotal role in regulating BA homeostasis. BAs are high-affinity endogenous FXR ligands. The order of potency of BAs is CDCA $>\mathrm{LCA}=\mathrm{DCA}>\mathrm{CA}$ (22). FXR activation regulates a network of genes in hepatic BA synthesis, biliary BA secretion, intestinal BA absorption and hepatic BA uptake $(22,23)$. The inappropriate function of FXR is often associated with liver diseases, including liver fibrosis and HCC, and previous studies have shown that FXR is deficient in liver cirrhosis $(24,25)$. The absence of FXR regulation is an important cause of BA disorders, and FXR agonists are considered to be potential medicines for BA regulation and cirrhosis treatment (24). On the basis of these factors, it is hypothesized that with the occurrence and progression of liver cirrhosis, the concentrations of individual BAs may correspondingly change.
This present study indicated that GCA, GCDCA, TCA, TCDCA, and TUDCA were significantly increased in patients with cirrhosis compared to patients without cirrhosis. These individual BAs may be potential indicators in the diagnosis of cirrhosis. In patients with progressive liver cirrhosis, the main alteration observed was a significant increase in conjugated primary BAs, whereas there was a decreasing trend in secondary BAs (DCA and LCA). A previous study also showed that progressive liver cirrhosis causes a decrease in the conversion of fecal primary BAs to secondary BAs (26).

Alterations in BAs are closely related to pathological changes during the development of cirrhosis. BAs synthesized in the liver are excreted in bile as conjugates with glycine or taurine; however, the secretion of BAs markedly diminishes in cirrhosis (27), which results in the intrahepatic cholestasis and the low concentrations of BAs in the intestine. Because the inhibitory effect of BAs on intestinal flora is weakened, intestinal bacteria overgrow $(5,28)$. BAs are rapidly deconjugated by bacteria and absorbed via non-ionic diffusion (29). In addition, with the progression of liver cirrhosis, portal hypertension leads to an increase in intestinal permeability $(29,30)$. This may increase the passive absorption of BAs via the plasma membrane, and further reduce the quantity of BAs entering the colon. Thus, conversion of primary BAs to secondary BAs by bacterial $7 \alpha$-dehydroxylase is decreased $(29,30)$. Therefore, with the development of cirrhosis, the level of total serum BAs is increased, especially conjugated primary BAs, which may be of great value to clinicians for diagnosing and evaluating the progress of liver cirrhosis.

BAs play an essential role in the deterioration of liver cirrhosis. Hydrophobic BAs, such as CDCA and GCDCA, are considered to be highly toxic (31). Accumulation of high levels 
A

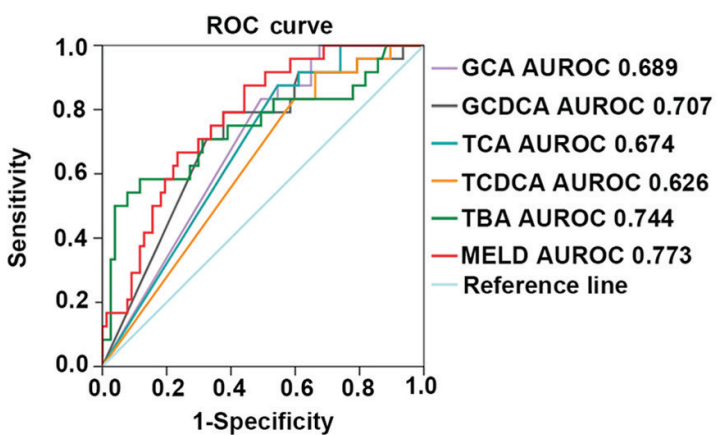

C

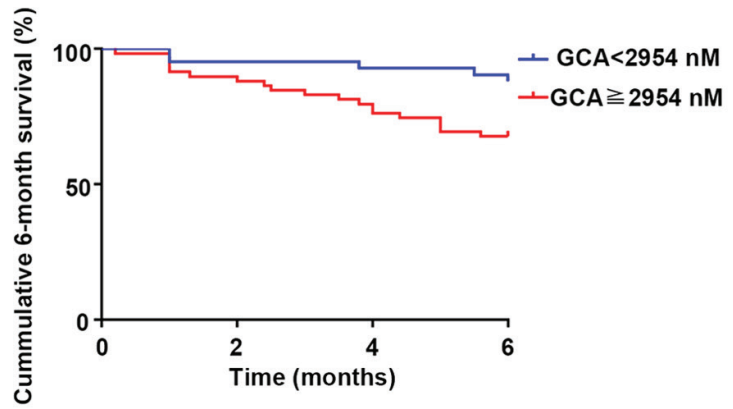

E

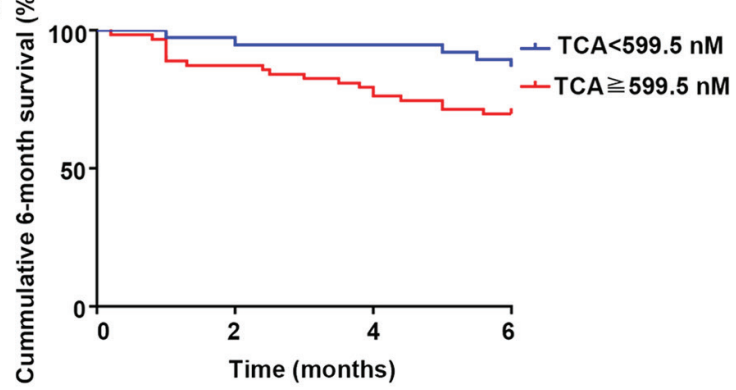

B

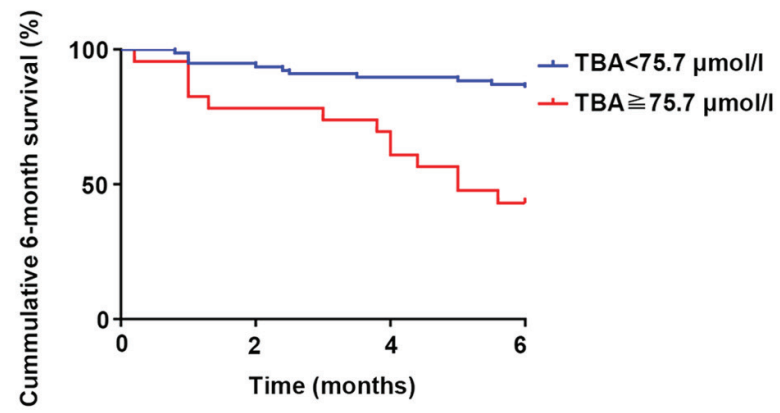

D

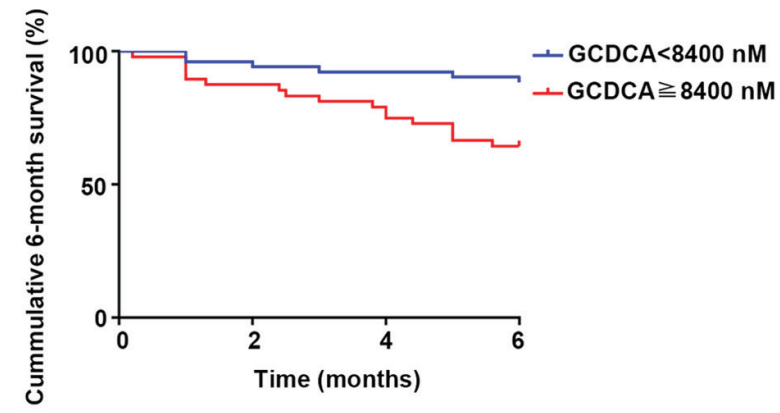

$\mathbf{F}$

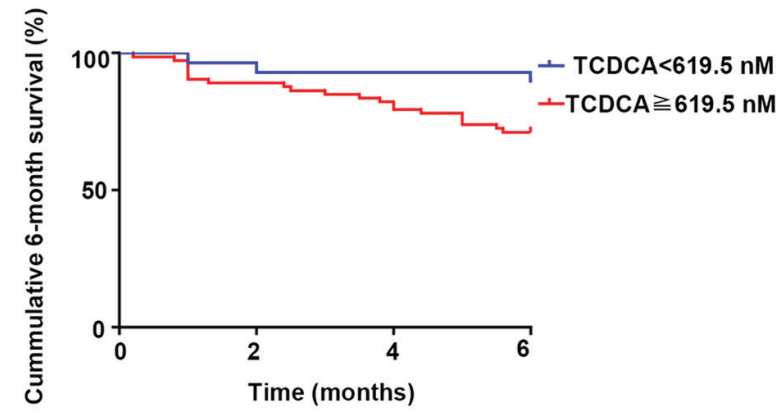

Figure 6. Survival analysis of TBAs and the four individual BAs. (A) The ROC curve of TBAs and four individual BAs in predicting the 6-month survival (TBA: $\mathrm{P}<0.001$; GCA: $\mathrm{P}=0.005$; GCDCA: $\mathrm{P}=0.002$; TCA: $\mathrm{P}=0.010$; TCDCA: $\mathrm{P}=0.064$ ). Kaplan-Meier plot of 6-month survival in patients with different levels of (B) TBAs, (C) GCA, (D) GCDCA, (E) TCA and (F) TCDCA (TBA: P<0.001; GCA: P=0.004; GCDCA: P=0.005; TCA: P=0.001; TCDCA: P=0.021). AUROC, area under the receiver operating characteristic curve; GCA, glycocholic acid; GCDCA, glycochenodeoxycholic acid; MELD, model for end-stage liver disease; ROC, receiver operating characteristic; TBA, total bile acids; TCA, taurocholic acid; TCDCA, taurochenoxycholic acid.

of hydrophobic BAs in hepatocytes may induce cell injury through promotion of inflammation and mitochondrial oxidative stress-mediated death pathways (7,32). Furthermore, the toxicity of BAs which have accumulated in the circulation is also reflected in organs other than the liver. Hydrophobic BAs are able to cross the blood-brain barrier, thus causing damage to the central nervous system and worsening the course of hepatic encephalopathy in animal experiments (33). Clinical studies have shown that BA retention is associated with hepatopulmonary syndrome and gas exchange abnormalities (34). BA overload has also been shown to be toxic to cardiomyocytes, thus inducing cardiomyopathy and metabolic dysfunction in the heart (35), and leading to life-threatening arrhythmias, vasorelaxation, and decreased peripheral resistance $(36,37)$.

Retention of toxic BAs may aggravate liver injury and influence the prognosis of cirrhotic patients. Total and individual BA values were further analyzed to examine their potential in predicting 6-month mortality among cirrhotic patients, which showed that total BAs, GCA, GCDCA and TCA were significantly altered in cirrhotic patients. The Kaplan-Meier plot suggested that the 6-month survival rate was significantly higher in patients with low levels of total and primary conjugated BAs. Notably, multivariate regression analysis showed that a high level of total BAs was an independent predictor of mortality in cirrhotic patients. The results showed that BAs are effective markers for predicting short-term survival in cirrhotic patients.

$\mathrm{HCC}$ is the most common cause of death in cirrhotic patients $(2,3)$. The lack of good biomarkers for early diagnosis of HCC may result in poor prognosis, especially for patients with compensatory liver function (38). Previous studies have shown that BAs are involved in the proliferation and regeneration of hepatocytes (39) and pathogenesis of hepatocellular carcinoma (40). Hydrophobic BAs may collaboratively promote liver carcinogenesis (41). In this present study, it was found that in patients with early cirrhosis, the concentrations of total BAs and primary conjugated BAs were significantly higher in patients who also had HCC compared to patients without HCC. This indicates that the occurrence of HCC should be considered in patients presenting with early cirrhosis and high levels of total and primary conjugated BAs. Total and primary conjugated BAs may be potential biomarkers for the early diagnosis of HCC in patients with early cirrhosis. 

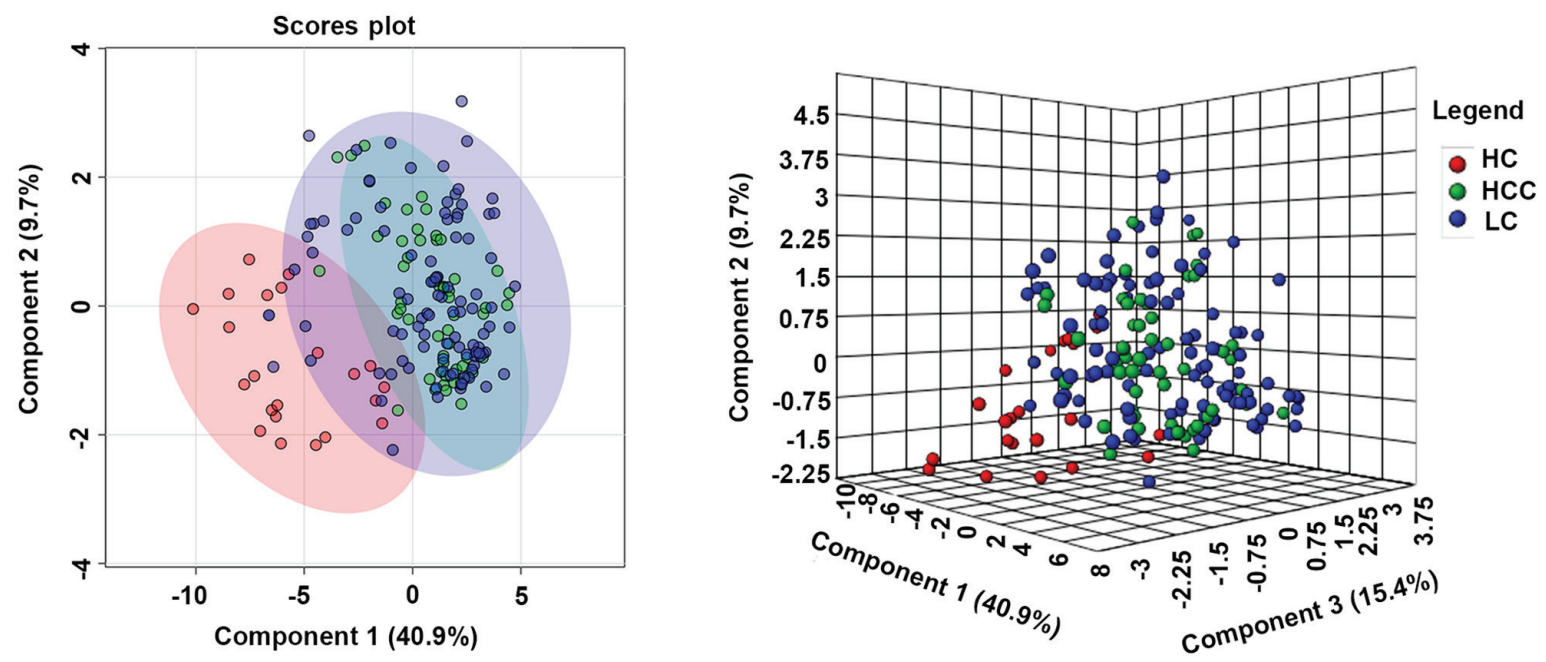

Figure 7. Two-dimension and three-dimension scores plots of partial least squares-discriminant analysis models of bile acid profiles. HC, healthy control; HCC, hepatocellular carcinoma; LC, liver cirrhosis.
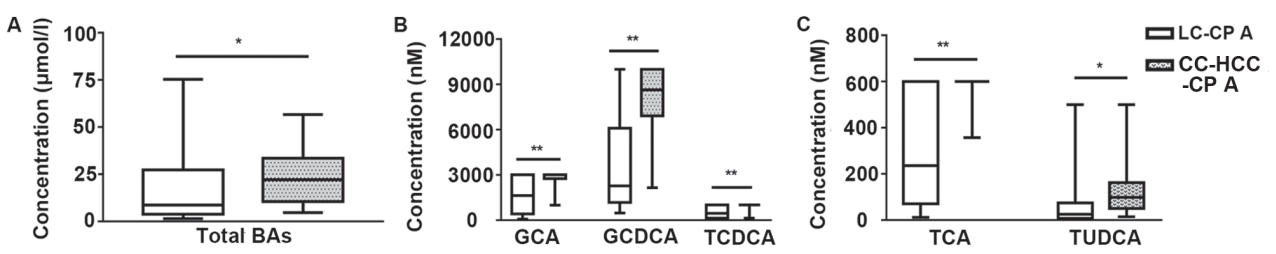

Figure 8. Box diagram of total BAs and five individual BAs in patients with cirrhosis and patients with CC-HCC in early-stage cirrhosis. (A) Comparison of total BAs. (B) Comparison of GCA, GCDCA, and TCDCA. (C) Comparison of TCA and TUDCA. "P $<0.05$, ${ }^{* *} \mathrm{P}<0.01$ (Mann-Whitney U test). BAs, bile acids; CC-HCC, cirrhosis complicated hepatocellular carcinoma; CP, Child-Pugh score; CP-A, early stage cirrhosis; GCA, glycocholic acid; GCDCA, glycochenodeoxycholic acid; HCC, hepatocellular carcinoma; LC, liver cirrhosis; TCA, taurocholic acid; TCDCA, taurochenoxycholic acid; TUDCA, tauroursodeoxycholic acid.

Taken together, the results of the present study verified the diagnostic value of the BA spectrum in liver cirrhosis. The results emphasized the close relationship between liver function and BA components, and suggested the prognostic role of BAs in cirrhotic patients; however, this present study also had some limitations. The sample was small and patients involved were all inpatients. The detection range was limited, which may increase the errors of the test to some extent. The limitation of the detection range is reflected in most detection methods. Although it was not possible to obtain exact values for the BA levels, they may still be used as potential diagnostic/prognostic markers of cirrhosis. Further investigations are needed to confirm this inference.

In conclusion, increased levels of total and primary conjugated BAs are associated with the occurrence, deterioration and prognosis of liver cirrhosis. High levels of total BAs may be an independent predictor of mortality in cirrhotic patients. Total and primary conjugated BAs may be potential biomarkers for the occurrence of $\mathrm{HCC}$ in patients with early cirrhosis.

\section{Acknowledgements}

Not applicable.

\section{Funding}

This study was financially supported by the Sustainable Development of Science and Technology Innovation Project of Chongming District, Shanghai (grant no. CKY2018-32) and Shanghai Natural Science Foundation (grant no. 19ZR1439900).

\section{Availability of data and materials}

The datasets used and/or analyzed during the current study are available from the corresponding author on reasonable request.

\section{Authors' contributions}

NL, JF, CG and YZ conceived and designed the experiments. $\mathrm{NL}, \mathrm{JF}, \mathrm{YL}$ and $\mathrm{QL}$ were responsible for data collection. NL, JD, and YX performed the data analysis. NL wrote the manuscript. JF and JD critically revised the manuscript before submission. All authors approved the final version of the manuscript.

\section{Ethics approval and consent to participate}

The Ethics Committee of Shanghai Tenth People's Hospital approved this study. Informed consent was obtained from all participants enrolled in the study.

\section{Patient consent for publication}

Not applicable. 


\section{Competing interests}

The authors declare that they have no competing interests.

\section{References}

1. Asrani SK, Devarbhavi H, Eaton J and Kamath PS: Burden of liver diseases in the world. J Hepatol 70: 151-171, 2019.

2. Fattovich G, Stroffolini T, Zagni I and Donato F: Hepatocellular carcinoma in cirrhosis: Incidence and risk factors Gastroenterology 127 (5 Suppl 1): S35-S50, 2004.

3. Forner A, Llovet JM and Bruix J: Hepatocellular carcinoma. Lancet 379: 1245-1255, 2012.

4. Boyer JL: Bile formation and secretion. Compr Physiol 3: 1035-1078, 2013

5. Arab JP, Martin-Mateos RM and Shah VH: Gut-liver axis, cirrhosis and portal hypertension: The chicken and the egg. Hepatol Int 12 (Suppl 1): S24-S33, 2018.

6. Meier PJ and Stieger B: Bile salt transporters. Annu Rey Physiol 64: 635-661, 2002.

7. Li T and Apte U: Bile acid metabolism and signaling in cholestasis, inflammation, and cancer. Adv Pharmacol 74: 263-302, 2015.

8. Wang H, Shang X, Wan X, Xiang X, Mao Q, Deng G and Wu Y: Increased hepatocellular carcinoma risk in chronic hepatitis $B$ patients with persistently elevated serum total bile acid: A retrospective cohort study. Sci Rep 6: 38180, 2016.

9. Mouzaki M, Wang AY, Bandsma R, Comelli EM, Arendt BM, Zhang L, Fung S, Fischer SE, McGilvray IG and Allard JP: Bile acids and dysbiosis in non-alcoholic fatty liver disease. PLoS One 11: e0151829, 2016

10. Wang X, Xie G, Zhao A, Zheng X, Huang F, Wang Y, Yao C, Jia W and Liu P: Serum bile acids are associated with pathological progression of hepatitis B-induced cirrhosis. J Proteome Res 15: 1126-1134, 2016.

11. Chen T, Xie G, Wang X, Fan J, Qiu Y, Zheng X, Qi X, Cao Y, Su M, Wang X, et al: Serum and urine metabolite profiling reveals potential biomarkers of human hepatocellular carcinoma. Mol Cell Proteomics 10: M110.004945, 2011.

12. Singal AK and Kamath PS: Model for end-stage liver disease J Clin Exp Hepatol 3: 50-60, 2013.

13. Kamath PS and Kim WR; Advanced Liver Disease Study Group: The model for end-stage liver disease (MELD). Hepatology 45 797-805, 2007.

14. Wiesner R, Edwards E, Freeman R, Harper A, Kim R, Kamath P, Kremers W, Lake J, Howard T, Merion RM, et al: Model for end-stage liver disease (MELD) and allocation of donor livers. Gastroenterology 124: 91-96, 2003.

15. Krautbauer S and Liebisch G: LC-MS/MS analysis of bile acids. Methods Mol Biol 1730: 103-110, 2018.

16. Chong J, Yamamoto M and Xia J: MetaboAnalystR 2.0: From raw spectra to biological insights. Metabolites 9: pii: E57, 2019.

17. Mannes GA, Thieme C, Stellaard F, Wang T, Sauerbruch T and Paumgartner G: Prognostic significance of serum bile acids in cirrhosis. Hepatology 6: 50-53, 1986.

18. Ohkubo H, Okuda K, Iida S, Ohnishi K, Ikawa S and Makino I: Role of portal and splenic vein shunts and impaired hepatic extraction in the elevated serum bile acids in liver cirrhosis Gastroenterology 86: 514-520, 1984.

19. Gilmore IT and Thompson RP: Plasma clearance of oral and intravenous cholic acid in subjects with and without chronic liver disease. Gut 21: 123-127, 1980.

20. Bajaj JS, Heuman DM, Hylemon PB, Sanyal AJ, White MB, Monteith P, Noble NA, Unser AB, Daita K, Fisher AR, et al: Altered profile of human gut microbiome is associated with cirrhosis and its complications. J Hepatol 60: 940-947, 2014.

21. Cassard AM and Ciocan D: Microbiota, a key player in alcoholic liver disease. Clin Mol Hepatol 24: 100-107, 2018.

22. Martinot E, Sèdes L, Baptissart M, Lobaccaro JM, Caira F, Beaudoin C and Volle DH: Bile acids and their receptors. Mol Aspects Med 56: 2-9, 2017.
23. Massafra V and van Mil SWC: Farnesoid X receptor: A 'homeostat' for hepatic nutrient metabolism. Biochim Biophys Acta Mol Basis Dis 1864: 45-59, 2018.

24. Kim SG, Kim BK, Kim K and Fang S: Bile acid nuclear receptor farnesoid X receptor: Therapeutic target for nonalcoholic fatty liver disease. Endocrinol Metab (Seoul) 31: 500-504, 2016.

25. Verbeke L, Farre R, Trebicka J, Komuta M, Roskams T, Klein S, Elst IV, Windmolders P, Vanuytsel T, Nevens F and Laleman W: Obeticholic acid, a farnesoid $\mathrm{X}$ receptor agonist, improves portal hypertension by two distinct pathways in cirrhotic rats. Hepatology 59: 2286-2298, 2014.

26. Kakiyama G, Pandak WM, Gillevet PM, Hylemon PB, Heuman DM, Daita K, Takei H, Muto A, Nittono $\mathrm{H}$, Ridlon JM, et al: Modulation of the fecal bile acid profile by gut microbiota in cirrhosis. J Hepatol 58: 949-955, 2013

27. Lorenzo-Zúñiga V, Bartolí R, Planas R, Hofmann AF, Viñado B, Hagey LR, Hernández JM, Mañé J, Alvarez MA, Ausina V and Gassull MA: Oral bile acids reduce bacterial overgrowth, bacterial translocation, and endotoxemia in cirrhotic rats. Hepatology 37: 551-557, 2003.

28. Seo YS and Shah VH: The role of gut-liver axis in the pathogenesis of liver cirrhosis and portal hypertension. Clin Mol Hepatol 18: 337-346, 2012.

29. Marin JJ, Macias RI, Briz O, Banales JM and Monte MJ: Bile acids in physiology, pathology and pharmacology. Curr Drug Metab 17: 4-29, 2015.

30. Gadaleta RM, van Mil SW, Oldenburg B, Siersema PD, Klomp LW and Van Erpecum KJ: Bile acids and their nuclear receptor FXR: Relevance for hepatobiliary and gastrointestinal disease. Biochim Biophys Acta 1801: 683-692, 2010.

31. Nuño-Lámbarri $N$, Barbero-Becerra VJ, Uribe $M$ and Chávez-Tapia NC: Elevated cholesterol levels have a poor prognosis in a cholestasis scenario. J Biochem Mol Toxicol 31: 1-6, 2017.

32. Gong Z, Zhou J, Zhao S, Tian C, Wang P, Xu C, Chen Y, Cai W and $\mathrm{Wu} \mathrm{J}$ : Chenodeoxycholic acid activates NLRP3 inflammasome and contributes to cholestatic liver fibrosis. Oncotarget 7: 83951-83963, 2016

33. Tripodi V, Contin M, Fernández MA and Lemberg A: Bile acids content in brain of common duct ligated rats. Ann Hepatol 11: 930-934, 2012.

34. Horvatits T, Drolz A, Rutter K, Roedl K, Fauler G, Müller C, Kluge S, Trauner M, Schenk $P$ and Fuhrmann V: Serum bile acids in patients with hepatopulmonary syndrome. Z Gastroenterol 55: 361-367, 2017.

35. Desai MS, Mathur B, Eblimit Z, Vasquez H, Taegtmeyer H, Karpen SJ, Penny DJ, Moore DD and Anakk S: Bile acid excess induces cardiomyopathy and metabolic dysfunctions in the heart. Hepatology 65: 189-201, 2017.

36. Pak JM and Lee SS: Vasoactive effects of bile salts in cirrhotic rats: In vivo and in vitro studies. Hepatology 18: 1175-1181, 1993.

37. Desai MS and Penny DJ: Bile acids induce arrhythmias: Old metabolite, new tricks. Heart 99: 1629-1630, 2013.

38. Cherqui D, Laurent A, Tayar C, Chang S, Van Nhieu JT, Loriau J, Karoui M, Duvoux C, Dhumeaux D and Fagniez PL: Laparoscopic liver resection for peripheral hepatocellular carcinoma in patients with chronic liver disease: Midterm results and perspectives. Ann Surg 243: 499-506, 2006.

39. Fan M, Wang X, Xu G, Yan Q and Huang W: Bile acid signaling and liver regeneration. Biochim Biophys Acta 1849: 196-200, 2015.

40. Altamirano-Barrera A, Uribe M, Lammert F and Méndez-Sánchez N: Bile acids and the risk for hepatocellular carcinoma in primary biliary cholangitis. Ann Hepatol 15: 453-454, 2016

41. Xie G, Wang X, Huang F, Zhao A, Chen W, Yan J, Zhang Y, Lei S, Ge K, Zheng X, et al: Dysregulated hepatic bile acids collaboratively promote liver carcinogenesis. Int J Cancer 139: 1764-1775, 2016

This work is licensed under a Creative Commons Attribution-NonCommercial-NoDerivatives 4.0 International (CC BY-NC-ND 4.0) License. 